\title{
Bone as a classic endocrine organ: Interactions with non-bone tissues
}

\author{
Elizabeth A. Streeten ${ }^{1}$
}

Published online: 5 July 2015

(C) Springer Science+Business Media New York 2015

In 1941, the term "postmenpausal osteoporosis" was coined by Fuller Albright [1]. Albright recognized that bone was a metabolically active tissue, in addition to serving an obvious structural role. In the 59 years that followed Albright's work, a great deal was learned about bone composition and metabolism, including the effects of many hormones on bone tissue, including parathyroid hormone and several steroid hormones (eg. calcitriol, estradiol, glucocorticoids). During this important time of accrual of knowledge on bone physiology, the effects of varied hormones on bone tissue was described in detail, but the affects of bone on other tissues was essentially unknown.

In 2000, ground breaking research from Ducy et al., revealed the brain-bone connection with their work on the effect of central nervous system leptin on bone metabolism [2]. This highly original research led to an explosion of studies on the interaction of bone with brain and with other non-bone organs including the pancreatic islets. Bone is now known to be an important regulator of energy metabolism. In the past 15 years, the affects of bone-derived hormones on non-bone tissue has clearly established bone as a bona fida endocrine organ. Proteins produced in the bone that perform true endocrine functions include osteocalcin and FGF23.

In this issue of Reviews in Endocrine and Metabolic Disease, the theme is "Bone as true endocrine organ". Important discoveries will be reviewed that support the endocrine function of bone. The first article is an overview of bone metabolism, by Gonciulea and Jan de Beur.

Elizabeth A. Streeten

estreete@medicine.umaryland.edu

1 Divisions of Endocrinology, Diabetes \& Nutrition and Genetics, University of Maryland School of Medicine, Baltimore, MD, USA
In the article "Metabolic functions of osteocalcin" Wei and Karsenty discuss their pioneering research that led to the discovery that bone metabolism is intertwined with energy metabolism through the production of osteocalcin (by osteoblasts). Osteocalcin is a key component of the regulation of glucose homeostasis through the bone-pancreas axis.

In the article reviewing the bone-brain axis, Chamouni, Schreiweis and Oury review the role of the brain in skeletal homeostasis, including the effect of adipocyte-derived leptin to activate neurons in the VMH (through serotonin) and sympathetic nervous system (SNS) to suppress bone formation. They further describe the role of the SNS in activating RANK $\mathrm{L}$ through adrenergic receptors on osteoblasts, leading to osteoclast recruitment and reduced bone formation. In opposition, the parasympathetic nervous system (Locus coeruleus (LC)) serves to upregulate bone mass. They further discuss the affect of osteocalcin to affect neurotransmitter production (including serotonin, NE, DA and GABA).

The ability of the bone to sense loading (exercise) and to set in motion changes that lead to improved bone strength is important for the prevention of fracture in the face of increased load. In the Mechanosensing article, Xiao and Quarles discuss two important mechanosensing pathways in cells of the osteblast lineage that sense flow within bone, involving genes polycystin 1 (PC1) and polycystin 2 (PC2) and sense changes in the bone microenvironment, involving TAZ (transcriptional coactivator with PDZ-binding motif, or WWTR1). These sensors act in concert to regulate the balance between osteoblastogenesis (through Runx2) and adipogenesis (through PPAR). Martin and Sims discuss the critical role and regulation of the RANKL/OPG pathway which regulates osteoclastogenesis.

Bone formation occurs through the activation of the canonical wnt pathway. Lara-Castillo and Johnson review the role of members of the LRP family (LRP4,5,6,8) of proteins in the activation of the wnt pathway. The authors discuss mutations 
in these genes that lead to human bone disease. Their discussion includes the role of sclerostin to inhibit the wnt signaling pathway. Sharifi, Ereifej and Lewiecki review research to date on sclerostin (produced by osteocytes) and its role as an endogenous antagonist to LRP5, resulting in inhibition of the wnt pathway.

In the Bone kidney interactions article, Nickolas and Jamal discuss recent advances in our understanding of chronic kidney disease-metabolic bone disease (CKD-MBD), formerly called renal osteodystrophy, including the importance of FGF23. In the final article, Blau and Collins review FGF23, a truly hormonal product of the osteocyte which serves a critical role in promoting renal $\mathrm{P}$ excretion.
I hope that this review will help the reader understand many important discoveries in bone metabolism that have resulted in the conclusion that bone is a true endocrine organ.

\section{References}

1. Albright F, Smith PH, Richardson AM. Postmenopausal osteoporosis clinical features. JAMA. 1941;116(22):2465-74.

2. Ducy P, Amling M, Takeda S, Priemel M, Schilling AF, Beil FT, Shen J, Vinson C, Rueger JM, Karsenty G. Leptin inhibits bone formation through a hypothalamic relay: a central control of bone mass. Cell. 2000;100(2):197-207. 